\title{
Coupled Deformation and Diffusion Process at the Stainless Steel/Carbon Steel Interface in the Deformation Bonding Process
}

\author{
S. Li, ${ }^{1}$ Q. D. Zhang, and J. Y. Liu
}

School of Mechanical Engineering, University of Science and Technology Beijing, Beijing, China

1 lishuoly@163.com

Cylindrical and surface-treated blanks were used to prepare stainless steel/carbon steel composite specimens at different temperatures. The coupled deformation and diffusion of materials at the interface were investigated by SEM and ultrasonic " $C$ " scanning detection. The difference in the elongation of the two metals is shown to result in tearing at the interface in the deformation bonding process. Cracks are mainly located in a more readily expanding substrate and are more pronounced at lower deformation temperatures. With an increase in deformation temperatures, the bonding efficiency also increases significantly, but the diffusion layer thickness is basically the same. The bonding efficiency of composites can be greatly improved by mechanical surface treatment at normal deformation temperatures.

Keywords: composites, interface, deformation bonding, diffusion, mechanical surface treatment.

Introduction. Over the past few years, bimetallic products, composed of two or more different materials, have been widely used in various industries due to their excellent properties [1]. The properties of metallurgical bonding transition zone (MBTZ) have a significant influence on the features of this kind of bimetallic products [2], which mainly determined by the condition such as thickness [3], arrangement and multiplicity of metals [4], surface preparation [5], bonding parameters [5,6] and post-heat treatment [7] of the composites. The formation of MBTZ is usually considered to be divided into three stages. In the first stage, close contact is produced in most areas of the interface under the yield and creep deformation mechanism. In the second stage, many voids are disappearing mainly by diffusion and grain boundary migration, while the migration of grain boundaries has left many remaining voids. In the third stage, the interface voids disappear entirely with the volume diffusion [8]. The mutual diffusion behavior of elements between different metals has been investigated broadly. To name a few, Lee [9] established the prediction model of the thickness of the diffusion layer of $\mathrm{Al} / \mathrm{Cu}$ and the hardness of the interface by the regression method. Jiao et al. [10] simulated the linear friction welding process of Ni/Al by molecular dynamics method and discussed the effect of surface morphology on atomic diffusion in interface zone Wang et al. [11] calculated the diffusion coefficient using the inverse method of Fick's second law.

However, there exist few reported studies on the deformation behavior of metal near the interface during the preparation process. Deformation bonding is the primary method for industrial production of bimetallic products, including the operations of pressing, rolling, explosive bonding, and extrusion [12]. In this study, deformation bonding at high and normal deformation temperature is conducted to explore the interactive deformation and diffusion of materials near the interface. The evolution law of the union rate and the influence of unequal extension between stainless steel and carbon steel on the MBTZ are investigated.

\section{Experimental Procedures.}

1.1. Deformation Bonding of Cylindrical Specimens. The commercial AISI 304 stainless steel and Q235A carbon steel were selected as raw materials, and the chemical compositions are given in Table 1. Five groups of samples were cut into cylindrical 
The Chemical Composition of AISI304 and Q235A (mass fraction/\%)

\begin{tabular}{||c|c|c|c|c|c|c|c|c||}
\hline Material & $\mathrm{Cr}$ & $\mathrm{Ni}$ & $\mathrm{C}$ & $\mathrm{Si}$ & $\mathrm{Mn}$ & $\mathrm{P}$ & $\mathrm{S}$ & $\mathrm{Fe}$ \\
\hline AISI 304 & 18.97 & 8.86 & 0.04 & 1.00 & 2.00 & 0.035 & 0.03 & Bal. \\
\hline Q235A & - & - & 0.22 & 0.30 & 0.43 & 0.040 & 0.05 & Bal. \\
\hline
\end{tabular}

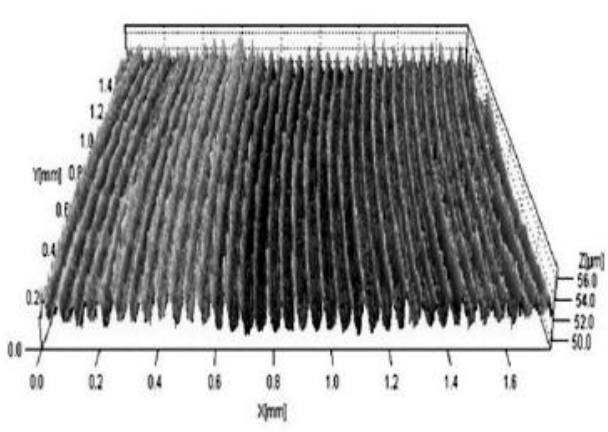

Fig. 1

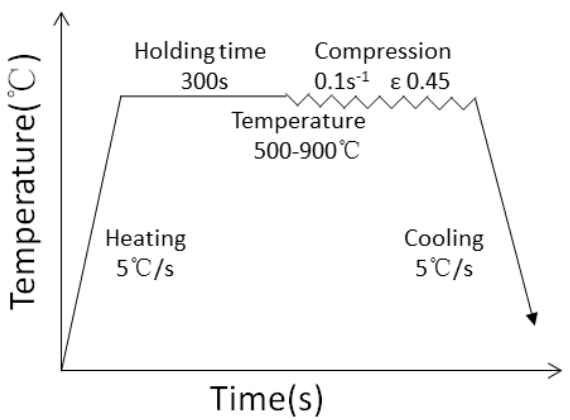

Fig. 2

Fig. 1. 3D figures of specimens' surfaces after grind.

Fig. 2. The experimental process.

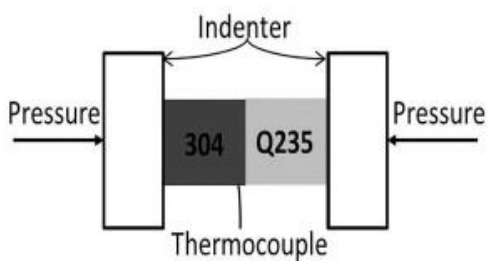

a

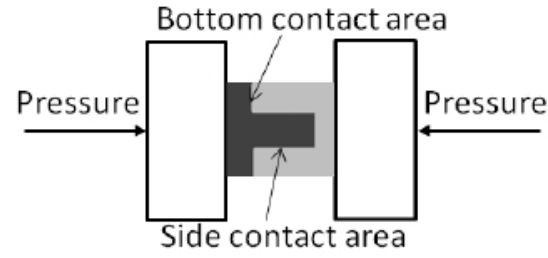

b

Fig. 3. The clamping method of (a) cylindrical specimens and (b) surface treated specimens.

specimens with a diameter of $8 \mathrm{~mm}$ and a length of $6 \mathrm{~mm}$. The surfaces of the specimens were treated by a grinding method, and the surface morphology is shown in Fig. 1. By using the Gleeble-3500 thermo-mechanical simulator, two kinds of materials were bonded together. As shown in Fig. 2, the specimens were firstly heated to the deformation temperature at a rate of $5{ }^{\circ} \mathrm{C} / \mathrm{s}$, and the heat preservation was $300 \mathrm{~s}$ to avoid the unequal heat of the specimens. The pressure bonding process was carried out in the temperature range of $500,600,700,800$, and $900^{\circ} \mathrm{C}$. The engineering strain rate was $0.1 \mathrm{~s}^{-1}$, and the corresponding engineering strain was $45 \%$. Tantalum sheets were selected to decrease the influence of mutual friction between indenter and sample in the hot uniaxial compression process. The clamping methods of cylindrical specimens are shown in Fig. 3a.

1.2. Deformation Bonding of Surface Treated Specimens. To study the influence of the different elongation of stainless steel and carbon steel on the interfacial bonding state during the deformation process. Two groups of stainless steel and carbon steel surface were processed with special mechanical treatment. In group 1, the surface of carbon steel was transformed into a boss shape, and the stainless steel surface was turned into a groove shape. In group 2, on the contrary, the surface of stainless steel was transformed into a boss shape, and the surface of carbon steel was processed into a groove shape. Each group of samples was chimed together for deformation bonding by using Gleeble3500 test machine 
under normal temperature. The engineering strain rate was $0.1 \mathrm{~s}^{-1}$, and the corresponding engineering strain was $45 \%$. The clamping methods of surface treated specimens are shown in Fig. 3b.

UST 200 immersion ultrasonic 'C' scanning detection system was used to detect the union rate of the bonding interface. Subsequently, all specimens were cut along the axis line, and the cross-section of the joints was ground and polished. The MBTZ, including the end contact zone of cylindrical specimens, the bottom and side contact zone of surface treated specimens (as shown in Fig. 3b), were carried out by scanning electron microscope (SEM) with energy dispersive spectrometer (EDS).

\section{Results and Discussion.}

2.1. Interface Union Rate of Joints Fabricated by Cylindrical Specimens. After thermal deformation bonding, the scanning images of the interface bonding state are shown in Fig. 4. According to the echo amplitude (AMP) visualized by ultrasonic ' $C$ ' scanning, different states can be distinguished through different colors. Blue regions represent the bonding states. Yellow regions represent the unbonded states. Green regions represent semi bonding states. The corresponding union rates of each specimen are 22.83,66.02, 100, and $100 \%$, respectively. It indicates that the effect of temperature on the union rate is obvious. No bonding zone exists between the interfaces at $500^{\circ} \mathrm{C}$. A certain degree of bonding occurs until $600^{\circ} \mathrm{C}$. With the increase of temperature, materials are easier to deform, and full integration of the interface can be achieved at $800^{\circ} \mathrm{C}$.

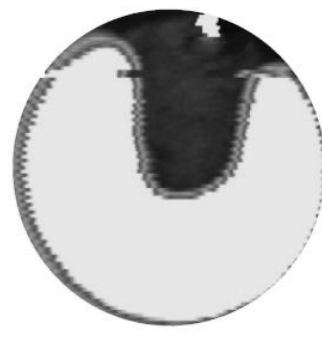

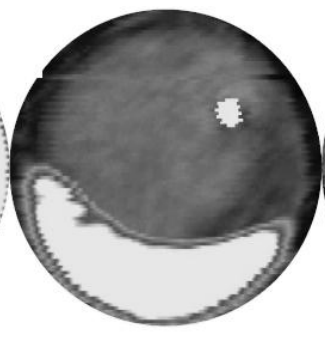

$\mathrm{b}$

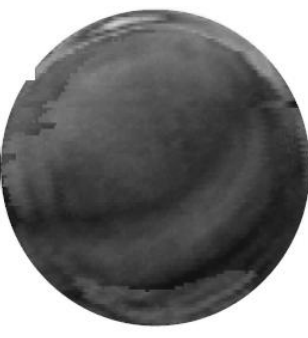

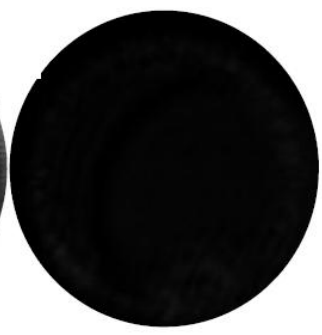

d

Fig. 4. Detection results of union rate at deformation temperature of 600 (a), 700 (b), 800 (c), and $900^{\circ} \mathrm{C}(\mathrm{d})$.

The metallurgical bonding over the entire interface does not co-occur. Owing to the influences of surface morphology and parallelism of the sample axis, the bonding first occurs somewhere on the interface. As the reduction increases, the real contact area between the metal interfaces increases gradually, and finally, the bonding region extends over the whole interface.

2.2. The MBTZ Fabricated by Cylindrical Specimens. The MBTZ in the middle and edge of the interface at $600^{\circ} \mathrm{C}$ are shown in Fig. $5 \mathrm{a}$ and $5 \mathrm{~b}$. Typically, the rough peaks between the interfaces begin to contact at the initial stage of the deformation. With the increase of the reduction rate, the roughness of the peak gradually begins to deform, and the actual contact zone increases continuously. It can be seen that discontinuous gaps still exist on the interface from Fig. 5a. As shown in Fig. 5b, a crack was found in the edge zone of the interface. EDS-profile shows that tearing occurs in the carbon steel matrix. This means that contact and inter-diffusion occur first during the process of deformation. Then the carbon steel part was torn apart because of different matrix elongation. The thickness of the diffusion layer, which defined as a region where the concentrations of carbon and stainless atoms both over $5 \%$, is about $2 \sim 3 \mu \mathrm{m}$ at the temperature of $600^{\circ} \mathrm{C}$.

The plastic deformation ability of the metal is enhanced as the deformation temperature rises to $700^{\circ} \mathrm{C}$. As shown in Fig. 6a, the width of the gap in the middle of 


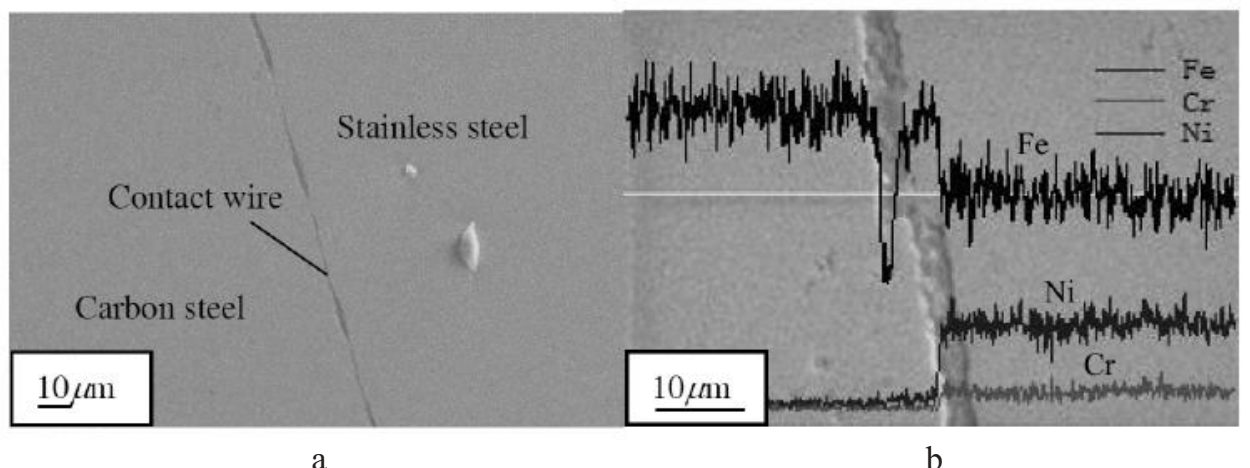

a

b

Fig. 5. The MBTZ close to center zone (a) and edge zone (b) at deformation temperature of $600{ }^{\circ} \mathrm{C}$.

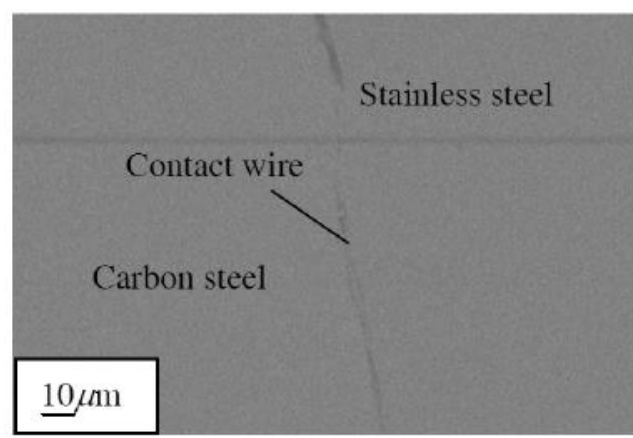

a

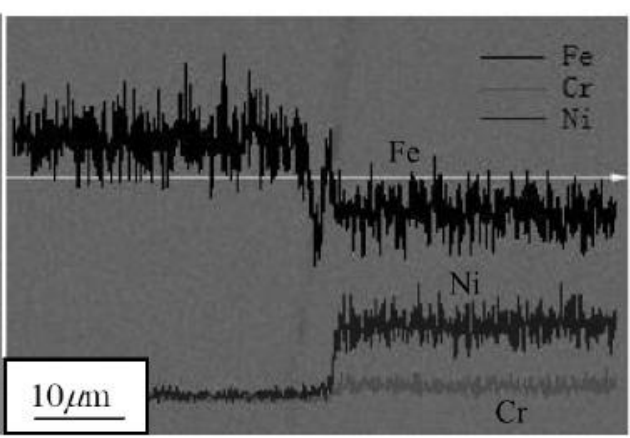

b

Fig. 6. The MBTZ close to center zone (a) and edge zone (b) at deformation temperature of $700^{\circ} \mathrm{C}$.

MBTZ is reduced. The tearing of carbon steel was still found at the edge zone of MBTZ from Fig. $6 \mathrm{~b}$, while the thickness of the diffusion layer is about $2 \sim 3 \mu \mathrm{m}$ at the temperature of $700^{\circ} \mathrm{C}$.

As shown in Fig. 7, with the increase of deformation temperature to 800 and $900^{\circ} \mathrm{C}$, the existence of cracks cannot be seen at the MBTZ, and the bonding quality is further improved. However, the width of the diffusion layer did not change significantly compared with 600 and $700^{\circ} \mathrm{C}$, indicating that the effect of deformation temperature on inter-diffusion behavior is not apparent.

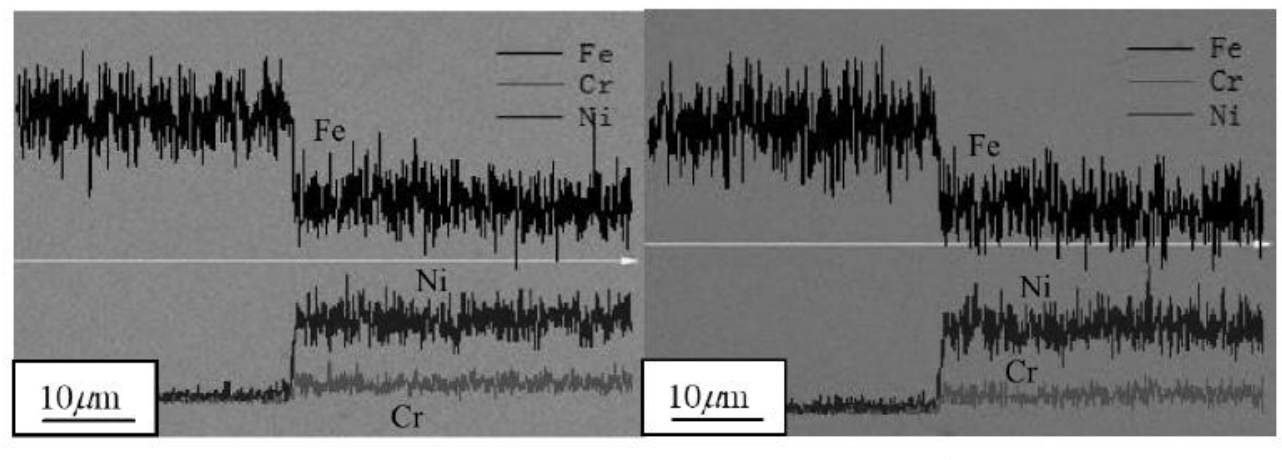

a

b

Fig. 7. The MBTZ at deformation temperature of 800 (a) and $900^{\circ} \mathrm{C}$ (b). 
2.3. The MBTZ Fabricated by Surface Treated Specimens. The MBTZ fabricated by surface treated specimens are shown in Fig. 8. For the group 1, the groove shape of stainless steel has a radial constraint on the boss shape of carbon steel in the process of deformation, which means that stainless steel is more likely to extend along the radial direction than carbon steel. Cracks are located on the side of the stainless steel matrix and around the entire interface. The thickness of the diffusion layer is about $2 \sim 3 \mu \mathrm{m}$ after deformation under the normal temperature from Fig. 8a. There is little difference in the thickness of the diffusion layer at high and low temperatures, which implies that the temperature is not the main factor affecting the diffusion. While the mutual contact time during the deformation process is very short, only shallow diffusion occurs at the interface between stainless steel and carbon steel.

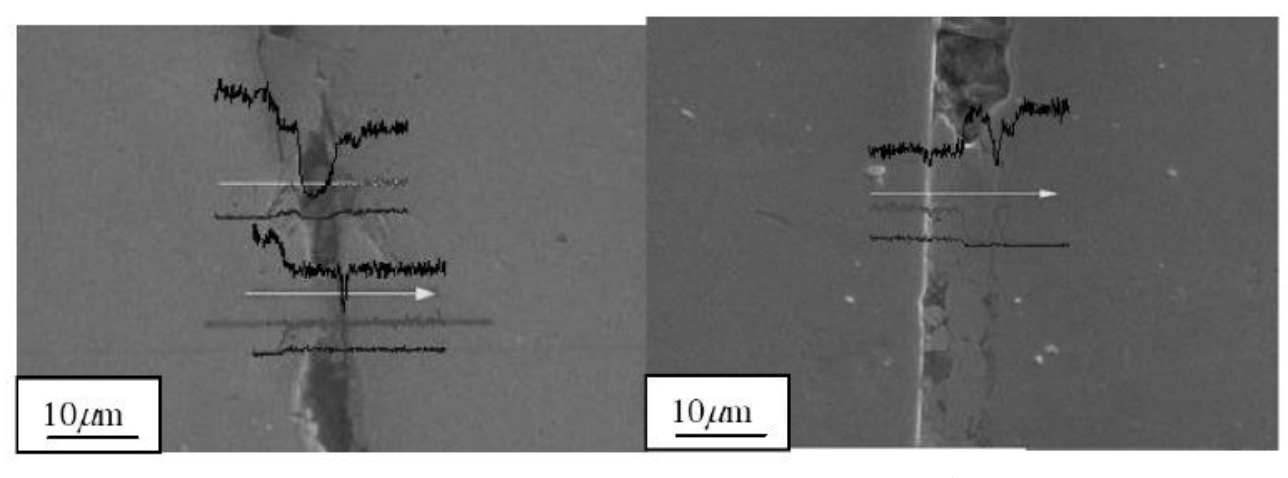

a

$\mathrm{b}$

Fig. 8. The MBTZ located in the bottom interface of group 1 (a) and group 2 treated by machinery (b).

Contrary to group 1, cracks were found in the carbon steel matrix in group 2. This is because the groove shape of carbon steel is more easy to extend and then fractured because of local stress concentration.

As shown in Fig. 9, there is no crack in the side contact zone of stainless steel and carbon steel matrix, which means the MBTZ has good bonding quality. A stable transition layer with a thickness of $2 \sim 3 \mu \mathrm{m}$ was found though line scanning. The difference in the elongation of bimetallic take disadvantage to the bonding in the bottom contact zone but promotes the bonding in the side contact zone. The interfacial bonding quality of bimetallic can be improved effectively by mechanical surface treatment.

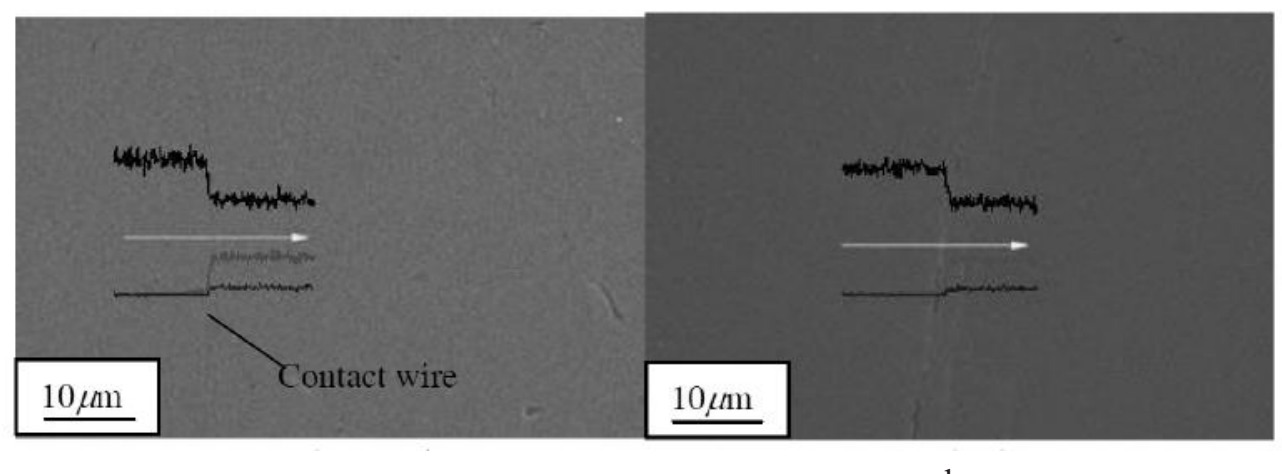

a

$\mathrm{b}$

Fig. 9. The MBTZ located in the bottom interface of group 1 (a) and group 2 treated by machinery (b). 
Conclusions. During the deformation bonding production of stainless-steel and carbon-steel joints, the deformation temperature has the most significant linear correlation with union rate but has little influence on the thickness of the diffusion layer. Only shallow diffusion can be realized, owing to the short contact time. Tear phenomenon may appear on the easily extended matrix as the deformation goes on. Moreover, the interfacial bonding quality can be improved by mechanical surface treatment.

Acknowledgments. This work was supported by the National Natural Science Foundation of China (No. 51575040).

1. J. Y. Jin and S. I. Hong, "Effect of heat treatment on tensile deformation characteristics and properties of Al3003/STS439 clad composite," Mater. Sci. Eng. A, 596, 1-8 (2014).

2. M. Knezevic, M. Jahedi, Y. P. Korkolis, and I. J. Beyerlein, "Material-based design of the extrusion of bimetallic tubes," Comput. Mater. Sci., 95, 63-73 (2014).

3. E. Y. Kim, J. H. Cho, H. W. Kim, and H. C. Shi, "Evolution of deformation texture in $\mathrm{Al} / \mathrm{Al}-\mathrm{Mg} / \mathrm{Al}$ composite sheets during cold-roll cladding," Mater. Sci. Eng. A, 530, 244-252 (2011).

4. I. K. Kim and S. I. Hong, "Effect of component layer thickness on the bending behaviors of roll-bonded tri-layered Mg/Al/STS clad composites," Mater. Design, 49, 935-944 (2013).

5. H. G. Kang, J. K. Kim, M. Y. Huh, and O. Engler, "A combined texture and FEM study of strain states during roll-cladding of five-ply stainless steel/aluminum composites," Mater. Sci. Eng. A, 452-453, 347-358 (2007).

6. R. Jamaati and M. R. Toroghinejad, "Investigation of the parameters of the cold roll bonding (CRB) process," Mater. Sci. Eng. A, 527, No. 9, 2320-2326 (2010).

7. R. Jamaati and M. R. Toroghinejad, "Effect of friction, annealing conditions and hardness on the bond strength of $\mathrm{Al} / \mathrm{Al}$ strips produced by cold roll bonding process," Mater. Design, 31, No. 9, 4508-4513 (2010).

8. V. R. Dave, I. J. Beyerlein, D. A. Hartman, and J. M. Barbieri, "A probabilistic diffusion weld modeling framework," Weld. J., 82, No. 7, 170-178 (2003).

9. T. H. Lee, Y. J. Lee, K. T. Park, et al., "Controlling Al/Cu composite diffusion layer during hydrostatic extrusion by using colloidal Ag," J. Mater. Process. Tech., 213, No. 3, 487-494 (2013).

10. Z. Jiao, C. Song, T. Lin, and P. He, "Molecular dynamics simulation of the effect of surface roughness and pore on linear friction welding between $\mathrm{Ni}$ and Al," Comput. Mater. Sci., 50, No. 12, 3385-3389 (2011).

11. T. M. Wang, F. Cao, P. Zhou, et al., "Study on diffusion behavior and microstructural evolution of $\mathrm{Al} / \mathrm{Cu}$ bimetal interface by synchrotron X-ray radiography," J. Alloy. Compd., 616, 550-555 (2014).

12. D. R. Lesuer, C. K. Syn, O. D. Sherby, et al., "Mechanical behaviour of laminated metal composites," Int. Mater. Rev., 41, No. 5, 169-197 (1996). 